\title{
Devices for accurate placement of epidural Tuohy needle for Anaesthesia administration
}

\author{
N. Vaughan ${ }^{1}$, V. N. Dubey ${ }^{1}$, M. Y. K. Wee ${ }^{2}$, and R. Isaacs ${ }^{2}$ \\ ${ }^{1}$ Bournemouth University, School of Design Engineering \& Computing, Bournemouth, UK \\ ${ }^{2}$ Poole Hospital NHS Foundation Trust, Poole, UK \\ Correspondence to: N. Vaughan (nvaughan@bmth.ac.uk)
}

Received: 17 April 2013 - Revised: 28 August 2013 - Accepted: 23 September 2013 - Published: 2 January 2014

\begin{abstract}
The aim of this project is to design two sterile devices for epidural needle insertion which can measure in real time (i) the depth of needle tip during insertion and (ii) interspinous pressure changes through a pressure measurement device as the epidural needle is advanced through the tissue layers. The length measurement device uses a small wireless camera with video processing computer algorithms which can detect and measure the moving needle. The pressure measurement device uses entirely sterile componenets including a pressure transducer to accurately measure syringe saline in $\mathrm{mm} \mathrm{Hg}$. The data from these two devices accurately describe a needle insertion allowing comparison or review of insertions. The data was then cross-referenced to pre-measured data from MRI or ultrasound scan to identify how ligemant thickness correlates to our measured depth and pressure data. The developed devices have been tested on a porcine specimen during insertions performed by experienced anaesthetists. We have obtained epidural pressures for each ligament and demonstrated functionality of our devices to measure pressure and depth of epidural needle during insertion. This has not previously been possible to monitor in real-time. The benefits of these devices are (i) to provide an alternative method to identify correct needle placement during the procedure on real patients. (ii) The data describing the speed, depth and pressure during insertion can be used to configure an epidural simulator, simulating the needle insertion procedure. (iii) Our pressure and depth data can be compared to pre-measured MRI and ultrasound to identify previously unknown links between epidural pressure and depth with BMI, obesity and body shapes.
\end{abstract}

\section{Introduction}

Epidural is commonly used as a form of pain relief during childbirth, for the treatment of chronic back pain or as a means to provide anaesthesia or analgesia during specific operations. The procedure consists of a long Tuohy needle being carefully inserted in the lumbar spine through layers of skin, tissue and ligament into the epidural space. Monitoring pressure and the needle depth during an epidural insertion is crucial because it indicates when the needle is placed precisely into the epidural space. If the needle is advanced too far it will puncture the dural sac and cause leakage of cerebrospinal fluid. Post dural puncture headaches may result and can be extremely disabling for the patient. Other risks include bleeding or nerve damage which may rarely lead to paralysis. If the needle is not within the epidural space, the pain relief may be ineffective or inadequate due to improper placement of the catheter.

The most important aspect of needle insertion is to judge the correct depth of the needle, because it has to pass through on average $42 \mathrm{~m}$ of tissue layers although this increases in obese patients, before arriving at the small $6 \mathrm{~mm}$ epidural space (Jenkins, 2005). This skill requires precise knowledge of the feeling as the needle passes through various tissue layers. The operator must judge the depth of the needle just from feeling. The point at which the needle arrives in epidural space is identified by a characteristic loss-of-resistance (LOR), with corresponding drop of pressure.

Standard epidural procedure starts by the anaesthetist inserting an $80 \mathrm{~mm}$ Tuohy needle through the skin and subcutaneous fat layer. A saline or air filled syringe is attached and the needle is slowly moved forwards through supraspinous 
ligament, interspinous ligament, ligamentum flavum and into the target epidural space. Each ligament has different density and texture, which affects the feel of the needle and the resistance to insertion (Vaughan et al., 2013). When the epidural space is reached, the resistance to insertion drops to zero and this indicates that the target has been reached so the device should show sudden drop in pressure. The depth can be precisely monitored from the pre-determined value from MRI or ultrasound scan for each patient before carrying out the procedure therefore ensuring the accurate placement of the needle.

Currently there is no way to electronically measure epidural needle depth or monitor epidural saline pressure for loss of resistance (LOR). These two novel devices developed in this paper provide solutions which can achieve these measurements in a sterile environment. The devices will provide several benefits to (i) provide a method alternative to operators opinion to confirm correct needle placement during an in-vivo procedure (ii) collect pressure and depth data from patients to incorporate into an epidural simulator (iii) crossreference measured data to MRI to identify how pressure and depth changes in patients with various BMI, tissue thicknesses and densities.

\section{Competing solutions}

Epidural needle insertion is essentially a 'blind' procedure, but utilizes a well-known technique referred to as "loss of resistance" (LOR). During an epidural insertion, the operator tries to perceive which tissue layer the needle tip is passing through by feeling the resistances on the needle. Current epidural precedure relies upon the operators judgement to identify LOR as the epidural needle travels through the various ligaments and potential spaces of the lumbar vertebral column. There are many risks involved with the epidural procedure due to its complexity. Since the actual insertion is 'blind' and the end-point cannot be visualized, the anaesthetist has only his/her perception from sensing and feeling of the needle resistance through the force stimuli in the fingertips and joints. There can be errors of judgment as to the location of the needle, exacerbated by individual patient characteristics of height, weight and body shape.

Our devices provide useful clues to the location of epidural needle which could be used in combination with operators judgement and may increase chance of successfully finding the epidural space. In our camera based depth measurement system, the needle depth is visually monitored using brown and silver markings on the needle shaft at $10 \mathrm{~mm}$ intervals. The manual method of depth monitoring may not be reliable since the anaesthetist has to pay attention to other aspects of the procedure such as pressure and angle of insertion, besides this it is believed that there may potentially be errors of $\pm 5 \mathrm{~mm}$ depending on the skill and perception of the anaesthetist. Attempts have been made in the past to measure the needle depth in real time by using metal calipers with an electronic sensor between the skin and the syringe barrel (Tran et al., 2009). Metal calipers are hard to sterilize for use in human trials and may interfere with the operator's standard procedure and would therefore not be ethically permitted in-vivo. Our novel sterile pressure measurement device captures the resultant pressures as the epidural needle is advanced through the tissue layers and the novel image processing technique which measures the insertion depth of the needle in real-time and transmits data wirelessly. Our pressure device can help to identify the sudden pressure drop at LOR, which identifies the needle is correctly in the epidural space, reducing risk of patient injury. Also the measured pressure data is very useful to (i) incorporate into a haptic epidural simulator and (ii) cross-reference data to pre-measured MRI and ultrasound to identify links between obesity and needle insertion pressure.

\section{Description of the design}

Both of our devices, the pressure monitor and the camera depth measurement device, include a novel wireless setup which has not been achieved before. The core component which enables our pressure device to obtain interspinous pressure measurements is a medical pressure transducer, ordinarily used for blood pressure and pulse monitoring, which is entirely sterile. This provides an electronic pressure reading, which we then amplify and this goes through a microcontroller, and is then transmitted wirelessly. The receiver can be located in a separate hospital room, which minimises disturbance, up to one mile away. Our method uses a novel three-way-tap, enabling the transducer to be placed between the needle and the syringe, so that any pressure changes will be detected by the device.

The purpose of using a camera for depth measurement is that the computer measures at a much faster and more accurate rate than human eye, and automating the process allows the operator to focus on their needle insertion. The realtime data describes insertion speed for later analysis. Also the computer could provide audio or visual cues when correct depth is reached. The camera based image-processing algorithms run on a laptop which can be in another hospital room to minimise disturbance. The wireless camera is placed within one metre of the needle insertion site on the patient. A full colour wireless camera is used. Received video signal was converted through USB for connection to a laptop. The laptop has custom algorithms which identify the depth of the needle by measuring the portion which is visible outside the skin. This length is subtracted from the full length of the needle to identify depth of needle tip beneath the skin. Algorithms achieve length measurement by colour detection of the blue handle and location of $10 \mathrm{~mm}$ silver markings which are a standard printed onto every epidural needle. The 
algorithm is custom made, based around a core of OpenCV library.

A wireless data transmitter is utilized to minimize the equipment and disruption in the labour room. This uses $\mathrm{XBee}^{\mathrm{TM}}$ Pro $60 \mathrm{~mW}$ with a PCB antenna at $2.4 \mathrm{GHz}$ frequency and $250 \mathrm{kbps}$ data transfer rate that allows a maximum of $100 \mathrm{~m}$ outdoor range. A computer receives the data remotely with a wireless receiver and displays a real-time graph on screen and simultaneously records the data to a file. The advantage of using a wireless transmitter is that the equipment is not only less cumbersome but also much less intrusive.

In obstetric practice, the LOR technique is performed with a saline-filled syringe connected directly onto the Tuohy needle, which maintains the plunger pressure until needle reaches the epidural space. Our design aims to minimize changes to this standard setup (Fig. 1). A small standard sterile three-way tap (BD Connecta ${ }^{\mathrm{TM}}$ ) is connected between the needle and syringe. The tap is connected to the pressure transducer via a one metre length of saline-filled sterile manometer tubing. The transducer's electrical plug is connected by a short electrical cable to the wireless transmitter box which measures only $12 \mathrm{~cm} \times 5 \mathrm{~cm} \times 3 \mathrm{~cm}$. The box contains a $5 \mathrm{~V}$ DC power supply, amplifier and the data transmitter. At the remote site, a wireless receiver is connected via Universal Serial Bus (USB) to the computer. Our custom designed software displays a real-time graph on screen and writes the data to a text file for later analysis; data retrieval speed can be varied in the software. The software clearly identifies loss-of-resistance as needle enters epidural space (Fig. 2) on the output from our pilot trial on a porcine cadaver. The data recorded into a file was plotted as a line graph shown in Fig. 3.

On needle depth measurement there were several problems associated with needle detection that makes measurement hard to accomplish: (i) the needle is a thin object with small width of only a few pixels; (ii) the needle is reflective stainless steel material which reflects colours from nearby objects, light sources and shadows; (iii) the needle is circular in cross section with different colours all around, the top is often illuminated while the underside is often shadowed; (iv) there are camera noise and blur from the wireless camera; (v) tilting the needle up/down causes colour change with only a small tilt of the needle; (vi) tilting towards/away from the camera causes the visible length to decrease, which may be confused with reduced length due to insertion; (vii) the needle is not the only object in the foreground, due to the operator's hands and the patient's back; (viii) lighting conditions vary from room to room; (ix) there is no time to configure the camera each time, due to the short notice given when an epidural is going to be performed and the anaesthetist cannot wait because the patient is often in pain; (x) the length is not fixed, but changes dynamically as needle is inserted. Solutions to these problems could involve controlling the environment with the use of fixed backgrounds, controlled light sources,

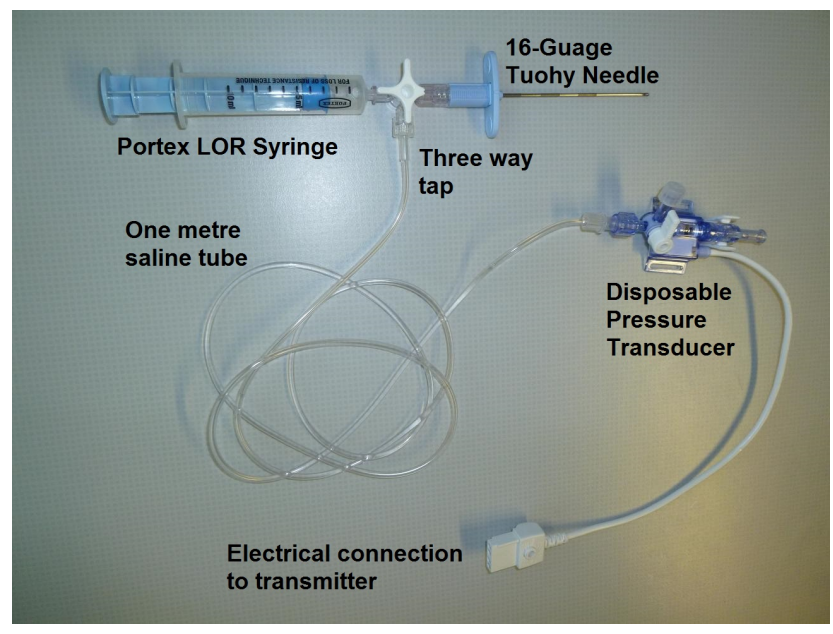

Figure 1. The disposable epidural pressure measurement system.

or restricting the movement. These precautions may not be feasible since they interfere with the operator's procedure and may not be ethically permissible. We developed custom software which detects the needle in the image, measures the length of the visible needle externally and transmits the depth data of the needle wirelessly in real-time. The algorithm design is able to solve the ten imaging problems listed by using the standard marking on the needle including blue handle and the silver $10 \mathrm{~mm}$ markings on the needle to accurately keep track of needle position, orientation and length in the majority of frames. The algorithmic implementation of the technique and the software output is shown in Figs. 4 and 5. This uses a wireless camera measuring $(20 \times 20 \times 20 \mathrm{~mm})$ manufactured by Ajoka that transmits a $640 \times 480$ pixel image as PAL AV in full colour over a $20 \mathrm{MHz}$ wireless link. The AV receiver is connected to a $2.6 \mathrm{GHz}$ Desktop PC with $2 \mathrm{~GB}$ RAM. The developed software uses features from OpenCV for image retrieval, memory handling and display.

\section{Evaluation and testing}

A porcine trial was conducted on a section of a cadaver for measurement of syringe pressure during insertion. The porcine tissue specimen was a double loin saddle cut. The cadaver was obtained from a livestock farm within 24 hours of slaughter without being frozen or modified in any way after slaughter to preserve the integrity of the spinal tissues. The pig was a standard hybrid Large White cross Saddleback. The specimen contained the entire back in one piece, with the whole spine, and all tissue layers from external skin, through to the thoracic cavity. The porcine tissue was mounted vertically against a wooden support to mimic sitting position so that the cadaver would not move when pressure was applied during insertion.

The camera and transmitter were set up one metre away from the site which mainly focused on the needle part, 


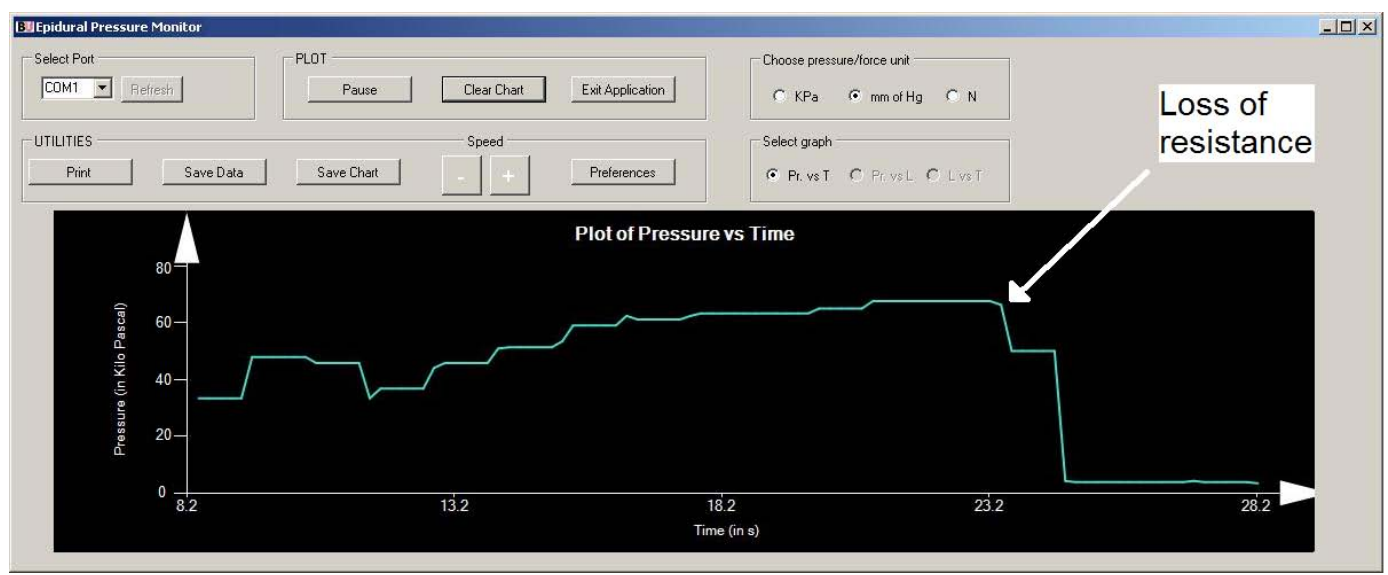

Figure 2. Software output clearly showing LOR.

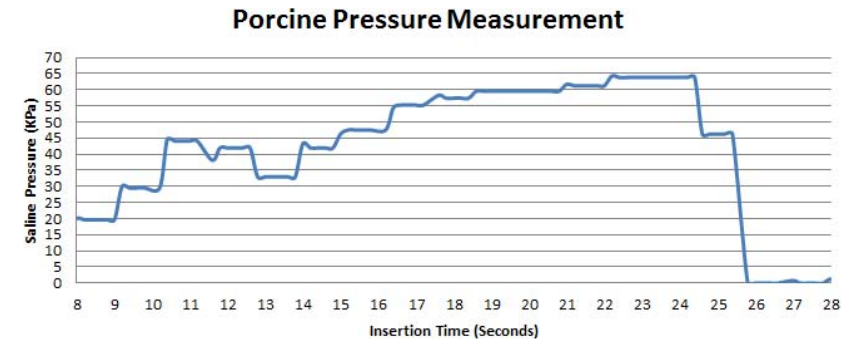

Figure 3. Line graph showing the KPa pressure measurement data.

therefore, needle patterns are easily discernible against the background. A saline drip bag was connected by tubing and flushed to fill the pressure transducer system. The vertical position of the transducer was compensated for the elevation difference between the transducer and the insertion point throughout to cancel any gravitational effect on the pressure readings. Epidural insertions were performed by two experienced anaesthetists.

The recordings of pressure were then started and continuously recorded throughout needle insertion until after the LOR had been experienced. The majority of insertions located the epidural space in the first attempt and the corresponding data is presented in Table 1, these values were averaged from all pressure values throughout each tissue and by the maximum pressure peak prior to LOR. Force was calculated from pressure, multiplying by the known area of the syringe barrel. Table 2 shows the mean value of the data collected and the standard deviation from this trial. This data compared quite well with similar previous studies (Holton, 2001; Tran et al., 2009), length data was verified by the actual measurement. The total insertion took about fifteen seconds with 10 frames per second. The maximum error from the camera based length measurement was $\pm 3 \mathrm{~mm}$ which was determined by comparison of each frame to a manual review of the video. During testing, failures were determined

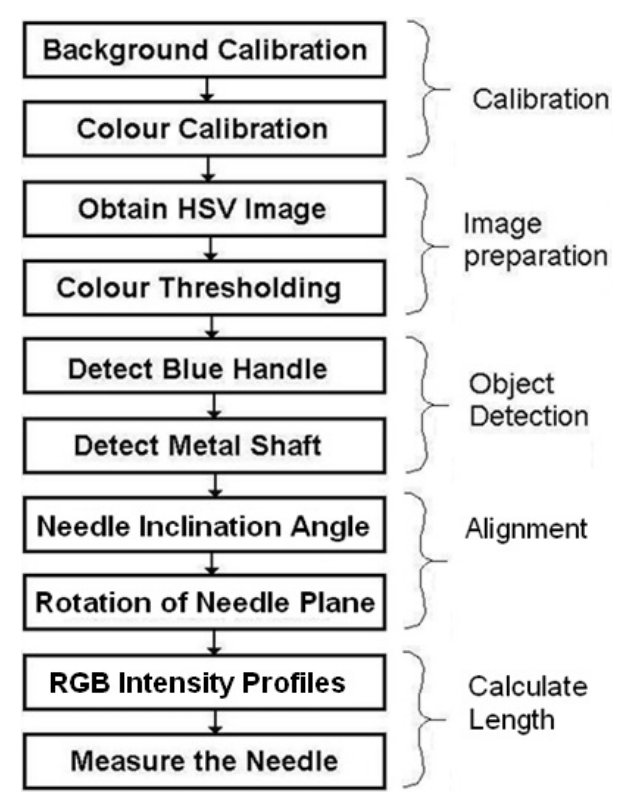

Figure 4. Implemented image processing algorithm.

as frames when needle markings were not identified by the algorithm so measurement did not work. The failure rate was 3 frames out of 150 which gave an overall $97.8 \%$ reliability during this test.

\section{Conclusions}

Using our device the data for pressure and depth were successfully captured during epidural insertions. The maximum pressure and depth measurements do match closely with the results from previous related studies. The results confirm correct functioning of our devices and the ability of this setup to be further used to take measurements from epidural administration in obstetric patients. The developed algorithm for measuring the depth of an epidural needle using the markings 


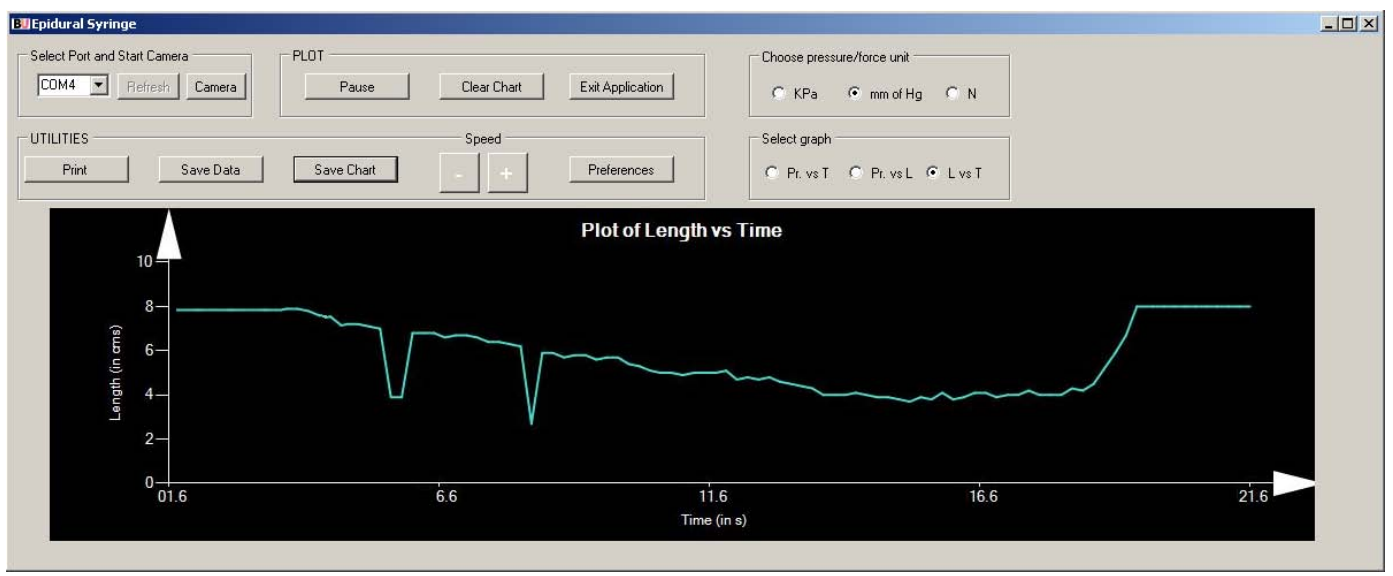

Figure 5. Software output measuring needle length.

Table 1. Force $(N)$ estimates for each tissue during needle insertions into porcine cadaver.

\begin{tabular}{lccccrcc}
\hline $\begin{array}{l}\text { Insertion } \\
\text { number }\end{array}$ & Skin & $\begin{array}{c}\text { Subcutaneous } \\
\text { fat }\end{array}$ & $\begin{array}{c}\text { Supraspinous } \\
\text { ligament }\end{array}$ & $\begin{array}{c}\text { Interspinous } \\
\text { ligament }\end{array}$ & $\begin{array}{r}\text { Ligamentum } \\
\text { flavum }\end{array}$ & $\begin{array}{l}\text { Epidural } \\
\text { space }\end{array}$ & Dura \\
\hline 2 & 11.5 & 5.3 & 7.5 & 8.5 & 11.4 & 0.0 & 0.9 \\
4 & 11.4 & 8.6 & 8.6 & 8.6 & 11.3 & 0.0 & 0.5 \\
5 & 11.3 & 7.1 & 9.4 & 8.2 & 9.0 & 0.5 & 0.8 \\
7 & 10.6 & 7.1 & 8.2 & 7.1 & 11.3 & 0.0 & 1.2 \\
10 & 10.6 & 8.2 & 9.4 & 8.5 & 10.8 & 0.0 & 1.4 \\
11 & 11.3 & 6.1 & 9.0 & 7.1 & 9.9 & 0.0 & 0.9 \\
\hline Mean with & 11.1 & 7.0 & 8.6 & 8.0 & 10.6 & 0.08 & 0.9 \\
(standard & $(0.37)$ & $(1.13)$ & $(0.67)$ & $(0.64)$ & $(0.88)$ & $(0.18)$ & $(0.28)$ \\
deviation) & & & & & & & \\
\hline
\end{tabular}

Table 2. Data from porcine trial (tissue thickness of each layer was measured at L2/L3).

\begin{tabular}{lcc}
\hline $\begin{array}{l}\text { Tissue } \\
\text { layer }\end{array}$ & $\begin{array}{c}\text { Tissue } \\
\text { thickness } \\
(\mathrm{mm})\end{array}$ & $\begin{array}{c}\text { Needle } \\
\text { depth } \\
(\mathrm{mm})\end{array}$ \\
\hline Skin & 3 & 0 \\
Subcutaneous fat & 6 & 3 \\
Supraspinous ligament & 4 & 9 \\
Interspinous ligament & 26 & 13 \\
Ligamentum flavum & 3 & 39 \\
Epidural space & 6 & 42 \\
Dura & 15 & 48 \\
\hline
\end{tabular}

on its metal shaft is clinically useful as there is currently no other way to measure the needle or monitor the depth in real-time during insertion. This could potentially be useful for precise location of Tuohy needle during epidurals to improve safety of the procedure. The image processing method is more ethically safe and less intrusive than using physical measuring devices which touch the needle (Tran et al., 2009). It also ensures sterility which is essential for the prevention of infection during epidural insertion. A further study with labouring women has now begun after obtaining ethical approval from the National Research Ethics Service in the UK, using our device and we hope that this will improve the safety of the procedure and thus reducing the morbidity and cost burden to the health services.

Acknowledgements. The authors thank Obstetric Anaesthetists Association for funding to continue conducting the trial with obstetric patients and MRI funding.

\section{Edited by: W. Durfee}

Reviewed by: two anonymous referees 


\section{References}

Holton, L. L.: Force models for needle insertion created from measured needle puncture data, Stud. Health Technol. Inf., 81, 180186, 2001.

Jenkins, J. G.: Some immediate serious complications of obstetric analgesia and anaesthesia: a prospective study of 145,550 epidurals, Int. J. Obstet. Anaesth., 14, 37-42, 2005.
Tran, D., Hor, K., Kamani, A., Lessoway, V., and Rohling, R.: Instrumentation of the loss-of-resistance technique for epidural needle insertion, IEEE T. Bio-Med. Eng., 56, 820-827, 2009.

Vaughan, N., Dubey, V. N., Wee, M., and Isaacs, R.: A review of epidural simulators: where are we today?, Med. Eng. Phys., 35, 1235-1250, 2013. 